\title{
Estrategia para Contribuir a la Implementación de la Formación Dual de los Profesionales de Ciencias Empresariales en las Pequeñas y Medianas Empresas de la Provincia Tungurahua, Ecuador
}

\author{
Ramiro P. Carvajal ${ }^{(1)}$, Ariel J. Romero ${ }^{(2)}$ y Gustavo Álvarez ${ }^{(2)}$ \\ (1) Universidad Técnica de Ambato. Campus Huachi. Avenida los Chasquis y Río Cutuchi. Ambato. \\ Tungurahua. Ecuador. (e-mail: ramiropcarvajal@uta.edu.ec) \\ (2) Universidad Regional Autónoma de los Andes (UNIANDES). Autopista vía a Baños Km 5 1/2 Ambato. \\ Tungurahua. Ecuador. (e-mail: arromero1970@yahoo.com)
}

Recibido Abr. 12, 2017; Aceptado May. 25, 2017; Versión final Jun. 12, 2017, Publicado Oct. 2017

\begin{abstract}
Resumen
El estudio presentado se realiza con la finalidad de proponer una estrategia para la implementación de la formación dual de los profesionales de las ciencias empresariales en las pequeñas y medianas empresas (PYMES) de la provincia Tungurahua, Ecuador. Se aplican encuestas a graduados de las carreras de Marketing y Gestión de Negocios y Organización de Empresas de la Facultad de Ciencias Administrativas para conocer el nivel de inserción laboral, así como el nivel de interacción con el sector empresarial en el transcurso de su carrera. Además, se encuestan empresarios de las PYMES de la provincia Tungurahua para conocer el nivel de satisfacción con la vinculación entre universidad y el sector productivo y las posibilidades de realizar en sus empresas la formación de los futuros profesionales vinculados a las ciencias empresariales. Se evidenció que los graduados se encuentran empleados pero no vinculados a su profesión y que la formación dual es beneficiosa tanto para la universidad como para el sector productivo.
\end{abstract}

\section{Strategy to Contribute to the Implementation of Dual Training of Professionals in Business Sciences in Small and Medium Size Enterprises in Tungurahua Province, Ecuador}

\begin{abstract}
The present study is carried out with the purpose of proposing a strategy for the implementation of the dual training of professionals of the business sciences in the small and medium size enterprises (SMEs) of the Tungurahua province, Ecuador. Surveys are applied to graduates of the Marketing and Business Management courses and Business Organization of the Faculty of Administrative Sciences to know the level of labor insertion, as well as the level of interaction with the business sector in the course of their career. In addition, businessmen from SMEs in the province of Tungurahua are surveyed to know the level of satisfaction with the linking university and productive sector and with the possibilities of doing in their companies the training of future professionals of the business sciences. It was evidenced that graduates are employed but not linked to their profession and that dual training is beneficial for the university and the productive sector.
\end{abstract}

Keywords: dual training; job placement; linking university company; competency-based training. 


\section{INTRODUCCIÓN}

La formación de profesionales competentes y comprometidos con el desarrollo socioeconómico de la sociedad, es una de las grandes prioridades de los sistemas de educación superior a nivel mundial y específicamente en el contexto latinoamericano. La UNESCO refuerza esta afirmación, al plantear en la Conferencia Mundial de Educación Superior de Paris 2009, que es necesario fortalecer las funciones de la educación superior al servicio de la sociedad en cuanto a los problemas prioritarios de la misma y el estrecho vínculo entre la universidad y el mundo del trabajo. (UNESCO, 2009). La vinculación de la universidad con el mundo del trabajo ha sido investigada por diferentes autores, entre los que sobresalen: (Velho, et al. 2010; Casalet, 2012; Montoya, 2012; Vicente, 2012; Mato, 2013; Arias, 2014; Quispe, 2014; Ruiz, 2015; Aguilar y Bermúdez, 2016; Marsollier y Aparicio, 2016, Novelo et al, 2016 y Rodríguez, 2016), entre otros. En sus trabajos se analiza la relación de la universidad con el sector empresarial y las oportunidades que deben aprovecharse desde los dos escenarios para desarrollar la investigación científica y la innovación tecnológica. Además, coinciden en que las universidades deben conocer las necesidades del sector empresarial para contribuir a solucionarlos, así como integrar a los futuros profesionales a la realidad del mercado laboral donde posteriormente se desempeñarán.

En el estudio de la relación educación y trabajo, (Vicente, 2012) plantea que existen dos tendencias fundamentales, una primera, que se centra en la relación de la pertinencia de la formación de los graduados con las necesidades y demandas reales del mercado laboral, y por tanto las competencias del graduado deben estar en función de las necesidades laborales que demanda el mundo del trabajo. Así mismo, se destaca una segunda corriente que defiende la teoría de que el análisis de la empleabilidad de los graduados debe enfocarse en las expectativas que ellos tengan sobre su campo profesional y laboral. En la actual sociedad del conocimiento, la relación de la universidad con el sector empresarial juega un papel importante, no obstante (Vega et al, 2011) reconoce que, a pesar de esa necesidad, la realidad evidencia que la incidencia de la universidad en el desarrollo local ha sido mínima, influenciada en mayor medida, por el desconocimiento que poseen las instituciones de educación superior de las necesidades del sector empresarial.

No obstante, la relación entre universidad y empresa se debe adecuar a las transformaciones que se producen en el entorno actual y tributar al cumplimiento de la misión de la educación superior de contribuir al desarrollo de la sociedad, mediante un proceso de aproximación permanente. En este sentido es importante que la universidad establezca dicha relación teniendo en cuenta que los intereses de las empresas se encuentren alineados con las necesidades de desarrollo mayoritarias de los grupos sociales en los cuales tienen incidencia. Se coincide con (Montoya et al, 2013), quien expone que la relación universidad - empresa debe garantizar que se formen adecuadamente sus estudiantes para la vida laboral, se transfiera conocimiento para el desarrollo económico y social, y las empresas perfeccionen sus procesos productivos, la eficiencia, la efectividad y la responsabilidad social.

La formación dual surge en Europa en la Edad Media, en esta modalidad el aprendizaje se realizaba bajo la supervisión de un maestro, el cual era responsable de la formación del joven en lo profesional y en lo educativo. Estos aprendices no recibían salario, pero se les cubrían todos los gastos hasta que cumpliera su aprendizaje y entonces a partir de ahí recibía su salario correspondiente. Las exigencias de la formación de profesionales cada vez son mayores, se necesitan respuestas a las trasformaciones socioeconómicas de la sociedad, lo cual devenga profesionales altamente calificados, preparados para dar respuestas a problemas cada vez más complejos e inciertos, por lo que el acercamiento a la realidad del mundo laboral puede ofrecerles herramientas para enfrentar esa realidad de manera competitiva.

La formación dual es una respuesta a las necesidades del mundo empresarial, a pesar de no estar muy generalizada en Latinoamérica. Esta es una propuesta académica muy innovadora que pretende el aprovechamiento de los recursos empresariales, los de las universidades y el aporte de los educandos en busca del aprovechamiento de las interacciones universidad, empresa y estudiante de manera cooperativa. De esta manera la formación dual se orienta a la formación integral mediante la alianza de la empresa y universidad, donde el estudiante logra adiestrarse en su profesión y prepararse mejor para ser competitivo en el mercado laboral. (Muñoz, 2008). Esta modalidad de formación no sólo es beneficiosa para el estudiante y la academia, también se convierte en una fortaleza para el sector empresarial la cual recibe una importante transferencia de conocimientos actualizados del estudiante y la institución educativa, lo que se convierte en importante fuente de ventajas competitivas al introducir los adelantos de la ciencia en función del perfeccionamiento de los procesos empresariales.

En el contexto mundial la formación dual ha sido analizada por autores como (Zholdasbekova et al, 2016; Adam et al, 2017; Marhuenda et al, 2017; Tokeran et al, 2017 y Yahaya et al 2017), exponen la experiencia de países como España, Malasia, Kazajistán, China y Francia, y aseguran que esta modalidad de formación 
a pesar de no ser tan nueva, no ha logrado incorporar a la mayor cantidad de organizaciones, empresas e industrias. Demuestran que las universidades deben continuar trabajando en la implementación de esta formación para garantizar la mejor preparación de los profesionales y el acercamiento a la práctica de la profesión.

El sistema de formación alemán, se convirtió en un referente mundial y específicamente para las experiencias previas desarrolladas en Latinoamérica, esto motivado por las características propias del sistema que se desarrolla en Alemania para la formación de los futuros profesionales. Este sistema se basa en una serie de principios como: la formación profesional para alcanzar mejores resultados económicos, sociales e individuales, competencias profesionales para un perfil técnico flexible y ajustado a la movilidad, aprendizaje fundamentado en la teoría y la práctica, formación profesional colegiada entre el Estado y la economía, financiamiento social de la formación profesional, y preparación del personal docente y formativo. Este modelo le ha permitido al mundo empresarial disponer de mano de obra bien preparada y a los profesionales poder insertarse de manera temprana en el mercado laboral.

En Latinoamérica se reconocen estudios realizados en diferentes países sobre la formación dual, son diversos los autores que han abordado el tema, entre ellos: (Muñoz, 2008; Coiduras et al, 2014; Llisterri et al. 2014; Hernández y Goe, 2015; Herrera et al. 2015; Carranza et al, 2016; Castillo et al. 2016; Jiménez, et al. 2016; y León, 2016), los cuales explican las experiencias de la formación dual en Argentina, Chile, Colombia, Costa Rica, Cuba y México, así como los aportes fundamentales que esta formación ha generado para los futuros profesionales. Latinoamérica no se ha destacado en el desarrollo de la formación dual, las experiencias son bastante diferentes y dependen mucho del contexto específico de cada país, esta modalidad de formación se ha implementado en los diferentes niveles educativos desde la secundaria hasta la universidad, tomando como ejemplo la experiencia el sistema dual alemán.

Kuppfer (2015) realiza un estudio sobre la formación profesional dual en Latinoamérica y expone las principales experiencias desarrolladas en la región, en su investigación realiza un levantamiento de las principales ofertas de profesiones duales que se estudian en algunos países de América Latina, demostrando que existe una amplia concentración en la enseñanza técnico profesional y en menor medida en la educación superior. En el contexto ecuatoriano, al igual que lo que ocurre en la región, se concentra la formación dual en los institutos tecnológicos superiores y las carreras que más presencia tienen son: Administración Industrial, Administración del Transporte de Carga, Administración de Empresas y Economía, Agricultura, Construcción, Biotecnología, Logística, entre otras. A nivel universitario existen pocas experiencias previas en la Universidad Católica de Cuenca, la Escuela Politécnica de Chimborazo y La Universidad Católica del Ecuador.

La Universidad Técnica de Ambato, ubicada en la provincia Tungurahua, cuenta en su estructura académica con la Facultad de Ciencias Administrativas, donde se estudian las carreras de Organización de Empresas y Marketing y Gestión de Negocios. Estas carreras poseen más de 40 años de creadas pero su relación con el mercado laboral, específicamente el sector empresarial no ha sido fructífero. Esto unido al problema de la inserción laboral de los graduados de la facultad, motiva el desarrollo de la presente investigación cuya finalidad es proponer las bases para la formación dual y el desarrollo de competencias del formando en las PYMES de la provincia Tungurahua, Ecuador.

\section{METODOLOGÍA}

La investigación desarrollada es predominantemente cualitativa, ya que se basa en el análisis de las causas que provocan la baja inserción laboral de los graduados universitarios de las carreras empresariales y la desvinculación de la academia con el sector empresarial de la provincia Tungurahua, con vistas a presentar las bases para la incorporación de la formación dual y el desarrollo de competencias del formando en las PYMES de la provincia de Tungurahua. Aunque se utilizan elementos cuantitativos para garantizar la representatividad del estudio y obtener información relevante sobre el problema estudiado. El estudio se realiza en la Facultad de Ciencias Administrativas de la Universidad Técnica de Ambato, se incluyen en el mismo los graduados de las carreras de Organización de Empresas y Marketing y Gestión de Negocios de los últimos 5 años, así como los empresarios de las PYMES de la provincia de Tungurahua.

\section{Recolección de los datos.}

Se aplicaron encuestas a los graduados de las dos carreras para conocer el nivel de inserción laboral, los requerimientos del mercado laboral para su desempeño y el nivel de interacción con el sector empresarial durante el desarrollo de su carrera. A los empresarios de las PYMES de la provincia Tungurahua, se les realizará una encuesta para conocer el nivel de satisfacción con la vinculación con la facultad, la valoración 
sobre la calidad de los graduados y las competencias que requieren desde el sector empresarial para los profesionales de la administración de empresas.

Población y muestra.

En el estudio se incluyen graduados de las carreras de la FCADM y empresarios de la provincia Tungurahua, para la determinación del tamaño de la muestra se sigue el siguiente procedimiento:

Graduados de las carreras

$n=\frac{Z^{2} * p * q * N}{e^{2}(N-1)+\left(Z^{2} * p * q\right)}$

Donde:

$Z$ : coeficiente que tiene en cuenta el nivel de confianza $(95 \%, z=1,96)$

p: probabilidad de éxito

$q$ : $(1-p)$ probabilidad de fracaso. Se asume el supuesto de máxima variabilidad estadística $p=q=50 \%$

e: margen de error (5\%)

$\mathrm{N}$ : tamaño de la población (cantidad de graduados de la Facultad de Ciencias Administrativas en los últimos 5 años igual a 521)

n: tamaño de la muestra

$n=\frac{1,96^{2} * 0,5 * 0,5 * 521}{0,05^{2}(521-1)+\left(1,96^{2} * 0,5 * 0,5\right)}$

$n=221$

Empresas a incluir en el estudio

La provincia Tungurahua posee registradas 1050 empresas distribuidas de la siguiente manera: Cámara de Comercio (901), Cámara de Industrias (64) y Cámara de la Pequeña Industria (85), para el cálculo del tamaño de la muestra se utiliza la misma ecuación que para los graduados con los mismos valores de los elementos que conforman la ecuación y se obtiene que deben ser incluidas en el estudio al menos 170 empresas, distribuidos de la siguiente manera: Cámara de Comercio (145), Cámara de Industrias (11) y Cámara de la Pequeña Industria (14), la muestra fue distribuida por afijación proporcional al tamaño de la población de cada estrato de empresas y serán escogidas por medio de un muestreo aleatorio sistemático.

Metodología empleada para el desarrollo de la investigación.

Paso 1. Fundamentación teórica sobre la formación dual: incluye el análisis de los conceptos, tendencias y principales fundamentos teóricos desarrollados por autores en el contexto nacional e internacional que permita conformar el estado del arte. Además, se analizan los antecedentes de la formación dual en la FCADM.

Paso 2. Análisis de la situación actual del mercado laboral: se presentan los elementos que definen las realidades del empleo en América Latina y el Caribe, presentando las estadísticas relacionadas con el empleo en la región, específicamente el empleo juvenil. También se realiza un estudio de inserción laboral de los graduados de ciencias empresariales de la FCADM, para lo cual se aplica una encuesta a graduados según el tamaño de muestra calculado.

Paso 3. Valoración de la calidad de los graduados: se aplica una encuesta a empresarios de la provincia Tungurahua, según el tamaño de muestra calculado para que evalúen la calidad de los graduados de ciencias empresariales que laboran en sus empresas, así como el nivel de formación de las competencias profesionales que poseen. 
Paso 4. Propuesta de estrategia para implementar la formación dual: se exponen las premisas, etapas y beneficios que aporta la estrategia para la implementación de la formación dual de los profesionales de las ciencias empresariales en las Pymes de la provincia Tungurahua. 


\section{RESULTADOS Y DISCUSIÓN}

Este acápite del artículo ha sido dividido en cuatro fases fundamentales, una primera relacionada con la fundamentación teórica sobre la formación dual, la segunda aborda situación actual del empleo en América Latina y la inserción laboral de los graduados de la Facultad de Ciencias Administrativas, una tercera que incluye los criterios que poseen los empresarios sobre la calidad de la formación de los graduados, y por último, la propuesta de estrategia para la formación dual de los profesionales de ciencias empresariales en las Pymes de la provincia Tungurahua.

\section{Fundamentos teóricos sobre la formación dual.}

El análisis de los fundamentos teóricos se inicia con los conceptos y terminología relacionados con la formación dual, los cuales se presentan a continuación:

\section{Conceptos y terminología.}

Vinculación universidad empresa: proceso mediante el cual se da una interacción entre las instituciones de educación superior y el sector empresarial. Está relacionada con la integración que debe existir entre la universidad y el sector empresarial en aras de garantizar el desarrollo socioeconómico de los grupos mayoritarios donde incide la universidad.

Inserción laboral de graduados: proceso de incorporación al trabajo de los graduados universitarios una vez concluido su período de estudios. Para que la inserción laboral sea efectiva, el graduado debe estar empleado vinculado a la profesión estudiada.

Formación dual: se conoce como un proceso de formación que se realiza entre una institución educativa, encargada de trasmitir conocimientos y la base teórica de la profesión, y una empresa o lugar de trabajo donde se tiene la posibilidad de aplicar la teoría en función de solucionar los problemas de la práctica, así como adquirir experiencia relacionada con la profesión.

Estrategia: conjunto de acciones interrelacionadas entre sí, cuya finalidad es contribuir a la solución de determinados problemas.

\section{Consideraciones teóricas sobre la formación dual.}

La formación dual es una modalidad de formación, que fundamenta el proceso enseñanza - aprendizaje en dos lugares diferentes, la institución formadora donde se lleva a cabo la formación teórica y práctica y una organización donde se desarrollan actividades productivas que complementan el proceso de formación de los profesionales. Se coincide con (Coiduras et al, 2014; Kupffer, 2015 y Adam et al 2017), quienes consideran que este proceso implica la vinculación de varios componentes del proceso de formación, los conocimientos teóricos, la formación en los diferentes escenarios de la institución educativa y el aprovechamiento de los recursos con que cuenta el medio laboral de la organización o empresa donde se complementa la formación.

En esta modalidad de formación, el estudiante es orientado por un tutor en la empresa, este tutor es una persona con un alto nivel de experiencia y conocimientos de la profesión. Esta persona se encarga de dar seguimiento a las actividades prácticas que realiza el estudiante en la empresa con vistas a desarrollar sus competencias, conjuntamente con un docente de la institución educativa que vela por el seguimiento metodológico del proceso de aprendizaje. (Hernández y Goe, 2015). Es importante que en la formación dual se dé respuesta a las necesidades reales del mercado laboral, por tanto, el eje fundamental de esta formación debe ser la empresa, motivado porque son los empresarios tienen un papel fundamental en la inserción laboral de los profesionales una vez que egresan de las instituciones educativas. La empresa, por tanto, debe participar activamente en la formación de los estudiantes y colaborar con la institución educativa para determinar la carga horaria, contenidos y actividades que el estudiante desarrollará en las instalaciones de la empresa.

En este proceso de formación se trata de que las personas adquieran competencias profesionales y comportamiento ético para colaborar con la solución de los problemas que pueden presentar las empresas, que a su vez se convierten en el posible futuro empleo de esos profesionales, una vez que egresen de la institución educativa. La formación dual contribuye de manera significativa al logro de los pilares básicos de la educación establecidos por la UNESCO: aprender a conocer, aprender a hacer, aprender a convivir juntos y con los demás, y aprender a ser. Este proceso de formación facilita el acercamiento del estudiante a la realidad del mercado laboral donde en futuro se desempeñará, el aprendizaje orientado al estudiante 
basado en la vivencia y contextualización de la práctica laboral y en función de las exigencias del contexto socioeconómico y de los avances tecnológicos. En el proceso de enseñanza aprendizaje se debe tener muy en cuenta las actitudes y aptitudes de los estudiantes, la identificación de sus necesidades de aprendizaje, así como la disposición y tecnologías de las empresas que se involucran en la formación.

La importancia de la formación dual es expuesta por diferentes autores (Vicente, 2012; Carranza et al, 2016; Zholdasbekova et al 2016; Adam et al, 2017 y Yahaya et al 2017), y hacen referencia a los siguientes elementos: es un proceso dinámico que contribuye a la adaptación del estudiante desde muy temprano al mundo laboral y el estudiante aprende de manera significativa debido a que no solo se apropia de la teoría sino que se vincula a la práctica; el estudiante se relaciona con los procedimientos, técnicas y tecnología que en la institución educativa muchas veces no posee, la institución educativa perfecciona sus planes de estudio incorporando las necesidades y realidades del mercado laboral, y la empresa perfecciona sus procesos por medio del aporte de los conocimientos y la innovación que proviene de la academia. Por tanto, la formación dual es beneficiosa tanto para la institución educativa, como para el mercado laboral.

\section{Realidades del empleo en América Latina y El Caribe.}

La realidad económica de América Latina y el Caribe en la actualidad se vuelve bastante compleja, según proyecciones de crecimiento la tendencia se inclina a la baja y en las previsiones realizadas por la Comisión Económica para América Latina y el Caribe (CEPAL) se registrará una contracción de alrededor del 0,5\%. Lo más alarmante en la actualidad, es que decrece la economía y se generan mucho menos empleos, aspecto que tiene una alta incidencia en la juventud. Según estimaciones de la Organización Internacional del Trabajo (OIT), en el año 2014 en América Latina y El Caribe se generaron un millón menos de empleos, esto implica que un número importante de personas está saliendo del mercado laboral. Esta baja participación, significa que una gran cantidad de personas dejarán de trabajar (sobre todo mujeres y jóvenes), por lo que no percibirán sus salarios y ello tendrá una influencia significativa en el bienestar familiar. Como resultado de esta desaceleración económica la tasa de desempleo subió $0,5 \%$ del año 2014 al 2015, es decir del 6,0\% del año 2014 al 6,5\% del año 2015, lo cual tiene un impacto negativo en las estadísticas de empleo de la región. (OIT, 2015).

En este contexto difícil e incierto, los jóvenes se encuentran en desventaja, pues el $40 \%$ de los desempleados de la región tienen como edad promedio de 15-24 años, y sus tasas de desocupación son entre 2-4 veces mayor que las personas adultas. A esto se une el crecimiento demográfico, aspecto que tiene una importante influencia en el empleo, se pronostica que, para los próximos 10 años, la región necesitará alrededor de 50 millones de empleos en función del crecimiento de la población en edad de trabajar. La realidad económica de América Latina y el Caribe para de la empleabilidad de graduados universitarios expone realidades muy complicadas. En el contexto ecuatoriano estudios realizados por (Álvarez,J. y Romero, A. 2015) evidencian que los graduados de Administración de Empresas de la Universidad Regional Autónoma de los Andes en varias ciudades del país tienen una baja tasa de inserción laboral (en algunos casos existe más de un $40 \%$ de graduados que no poseen empleo), aspecto que demuestra que Ecuador no está ajeno a la realidad latinoamericana.

\section{Estudio de inserción laboral de los graduados de la Facultad.}

Con el objetivo de conocer el nivel de inserción laboral de los graduados, sus valoraciones sobre la preparación recibida para su inserción en el mercado laboral y sus criterios sobre la calidad de la formación, se aplican encuestas a 244 graduados al finalizar el semestre abril- septiembre del año 2015. Estas encuestas se aplican a través del sistema de seguimiento a graduados de la facultad.

1. El $72 \%$ de los graduados se encuentran en estos momentos empleados, sin embargo, menos del $40 \%$ de estos graduados que trabajan lo hacen vinculados a su profesión, lo cual evidencia que, aunque se encuentran empleados una gran mayoría de los graduados no lo hacen relacionado con la profesión estudiada.

2. La mayoría de los graduados $(72,7 \%)$ encuentran empleo por medio de la gestión personal, preferiblemente a través de contactos personales. El 76,3\% de los graduados percibe ingresos hasta 2 salarios básicos como remuneración de su trabajo, lo cual manifiestan le contribuye a solventar las necesidades básicas.

3. Sólo el $20 \%$ de los graduados han creado emprendimientos, el $18 \%$ trabaja en el sector público y el restante $62 \%$ lo hace en la empresa privada.

4. El $55 \%$ de los graduados manifiesta que la relación con el sector empresarial en la carrera no es suficiente para la posterior inserción laboral. Los graduados manifiestan que los dos únicos momentos de 
interacción con el sector empresarial son las prácticas preprofesionales y las tesis, por lo que no se aprovecha el transcurso de la carrera para establecer vínculos entre la universidad y el sector empresarial.

5. El $94,3 \%$ de los graduados considera que sería muy útil en su formación y para la posterior inserción en el mercado laboral desarrollar la formación dual en las carreras, por lo que reconocen la utilidad de la formación dual en la preparación de estos profesionales.

De estos resultados se puede inferir que existe un bajo nivel de inserción laboral de los graduados de las ciencias empresariales en la provincia Tungurahua, que, además, la mayoría de los graduados que trabajan lo hacen vinculados a otras actividades que no tienen relación con la profesión estudiada. La bolsa de empleo como mecanismo de facilidad y ayuda a la búsqueda de empleo de los graduados no está contribuyendo con su finalidad, y la relación universidad-empresa en el proceso de formación de la carrera es insuficiente en la preparación de los graduados para el mercado laboral.

Criterios de los empresarios sobre la calidad de los graduados.

Se aplica una encuesta a 170 empresarios de las PYMES de la provincia Tungurahua para conocer sus criterios sobre la vinculación de la universidad con el sector empresarial, la calidad de los graduados de las carreras de la Facultad de ciencias Empresariales, las competencias que deben tener estos graduados y el nivel de aceptación con la implementación de la formación dual.

1. El $60,7 \%$ de los empresarios encuestados manifiesta conocer las carreras de Organización de Empresas (OE) y Marketing y Gestión de Negocios (MGN) de la Facultad de Ciencias Empresariales de la UTA. De los 103 empresarios que conocen las carreras de la facultad, sólo el 58,5\% manifiesta que en su empresa se han realizado actividades relacionadas con dichas carreras.

2. Las acciones que más se han desarrollado entre las carreras de OE y MGN y el sector empresarial son: prácticas preprofesionales (78\%), pasantías (35\%) y tesis $(27 \%)$.

3. El criterio sobre la calidad de los graduados de las dos carreras de la FCADM se refleja en la figura 1, en ella se muestra que más del $50 \%$ de los empresarios considera que la calidad de dichos graduados no es buena.

4. En la figura 2 se presentan las competencias que consideran los empresarios deben tener los graduados de las carreras vinculadas a la administración de empresas para un buen desempeño en el sector empresarial. Como se puede observar en la figura 2, los empresarios consideran que las competencias más importantes para el buen desempeño en el mercado laboral de los graduados de estas carreras son: capacidad para la toma de decisiones, pensamiento estratégico, liderazgo, trabajo en equipo, administración y desarrollo del talento humano y capacidad para responder bajo presión.

5. En relación a la utilidad de la formación dual para la empresa y la universidad, el $87,1 \%$ de los empresarios consideran que es muy útil y manifiestan su disposición para contribuir a la implementación de la misma es sus empresas, lo que demuestra que existe la disposición de los empresarios para la implementación de esta modalidad de formación.

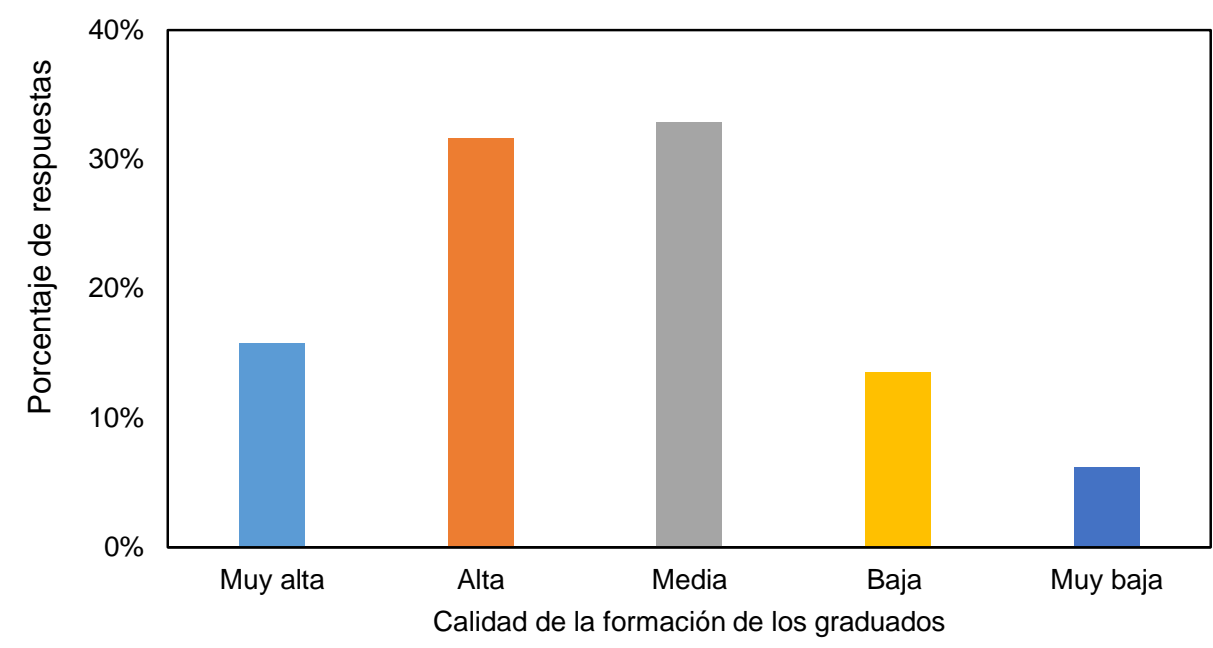

Fig. 1: Valoración sobre la calidad de los graduados de las carreras OE y MGN por parte de los empresarios 


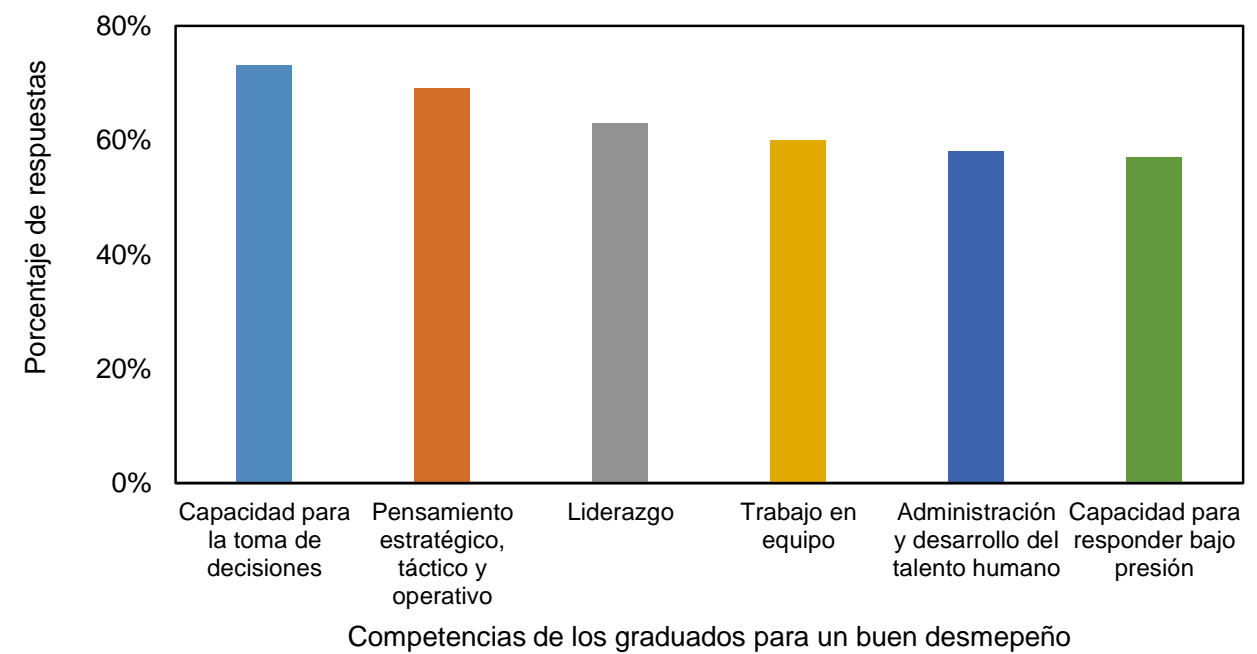

Fig. 2: Valoración sobre las principales competencias que deben tener los graduados para un buen desempeño

Estos resultados demuestran que, aunque la mayor parte de los empresarios conocen la FCADM, sólo la mitad de ellos han realizado acciones de vinculación con las carreras que en ella se imparten. Dentro de las acciones que se han desarrollado sólo se mencionan prácticas, pasantías y tesis, lo cual evidencia que es limitada la implementación de la formación dual. Los empresarios consideran que la calidad de los graduados no es buena y que las competencias que deben tener para la inserción en el mercado laboral están relacionadas con la toma de decisiones, capacidad de pensamiento, liderazgo, trabajo en equipo y respuesta bajo presión. De estas competencias, solamente las relacionadas con toma de decisiones y trabajo en equipo se encuentran dentro del perfil de egreso de las carreras de la facultad, por lo que se hace necesaria la actualización de este perfil en dichas carreras para que tributen a las necesidades del mercado laboral. Por último, los empresarios manifiestan que la formación dual sería muy útil para sus empresas y están dispuestos a participar en el proceso de formación, fortaleza que se debe aprovechar para su implementación

\section{Propuesta de implementación de la formación dual en las PYMES.}

La estrategia propuesta para la implementación de la formación dual para los estudiantes de ciencias empresariales en las PYMES de la provincia Tungurahua se fundamenta en las siguientes premisas:

1. Se desarrolla bajo un esquema de compromiso y responsabilidades compartidas entre las PYMES y la Facultad de Ciencias Administrativas de la UTA, es decir, que el proceso de formación se lleva a cabo en la universidad y la empresa.

2. Se definen monitores en las PYMES los cuales deben ser asesorados por los docentes de la carrera para el acompañamiento de los estudiantes en el proceso de formación en la empresa.

3. Se debe diseñar el proceso de formación de manera tal que se cumplan los objetivos de aprendizaje de la carrera y las empresas utilicen los conocimientos generados en este proceso de aprendizaje para la innovación en la gestión de sus procesos.

\section{Etapas de la estrategia.}

Las etapas de la estrategia están relacionadas con las fases de la formación dual en el contexto universidad mercado laboral y tienen la finalidad de contribuir a la implementación de esta modalidad de formación en las Pymes de la provincia Tungurahua.

Primera etapa. Antecedentes de la formación dual en la provincia Tungurahua.

La experiencia previa en la facultad de la formación dual es prácticamente nula, el modelo educativo que se emplea actualmente y desde hace más de 10 años, se basa en el constructivismo como tendencia pedagógica contemporánea. Los estudiantes permanecen los cinco primeros semestres de la carrera recibiendo clases solamente en las aulas, por lo que su acercamiento a la realidad del mundo empresarial es muy limitado. Posteriormente en los semestres seis, siete y ocho realizan prácticas preprofesionales en las empresas de la provincia, no obstante, esta práctica no es bien orientada, pues en la mayoría de los casos el estudiante desarrolla actividades no vinculadas a su perfil profesional, y no se cuenta con una planificación detallada de los objetivos de aprendizaje del proceso de prácticas. 
En este ámbito es importante señalar que la mayoría de las empresas de la provincia Tungurahua son micro y pequeñas, fundamentalmente negocios familiares, con un bajo nivel de innovación y que su período de supervivencia no supera los tres años. Esto unido a que los esfuerzos desarrollados por la facultad para el acercamiento al sector empresarial han sido limitados, han impedido que se pueda desarrollar la formación dual de los profesionales de las ciencias empresariales de manera efectiva en la provincia.

Segunda etapa. Identificación de las necesidades de las dos organizaciones: es vital en esta etapa poder integrar el interés de las PYMES de la provincia con las necesidades de formación de las carreras de la Facultad de Ciencias Empresariales (FCADM). Es importante la integración creativa y dinámica de los dos actores (PYMES y FCADM) para lograr resultados satisfactorios. La facultad trabaja en la creación de un Centro de Transferencia de Tecnología para viabilizar la relación con el sector empresarial y se encuentra en un proceso de rediseño curricular de las carreras que incluirá estos elementos. Además se han involucrado a los empresarios de la provincia en el diseño de los planes de estudios de las carreras para la formación dual, también se han desarrollado acciones de integración con el gobierno municipal y provincial y los gremios de los sectores más importantes del territorio (Cuero y calzado, textil, industria metalmecánica, agricultura, ganadería, mercado mayorista de distribución de mercancías, industria del plástico, cámara de turismo, etc)

Tercera etapa. Implementación curricular: A partir de la identificación de las necesidades de los dos actores en el proceso de formación dual, se debe contribuir a la satisfacción de esas necesidades desde dos líneas fundamentales.

i) se debe desarrollar la formación por módulos, estructurando los contenidos de acuerdo a las consideraciones de las áreas académicas de cada carrera, lo cual contribuye a establecer una relación estrecha entre unidades y asignaturas dentro de la cadena de valor curricular. Es muy importante definir las competencias del formando en función de las necesidades del mercado laboral. En el proceso de rediseño se ha modificado el perfil de egreso, en función de las competencias establecidas en el Proyecto Tunning Latinoamérica y las competencias que el sector empresarial ha propuesto como más importantes y que hoy no están contempladas (Capacidad para la toma de decisiones, pensamiento estratégico, táctico y operativo; liderazgo; administración del talento humano, capacidad para la solución de problemas, trabajo en equipo, compromiso con la calidad, capacidad para responder bajo presión, identificar y administrar riesgos de negocios, administrar un sistema logístico integral, gestionar sistemas de control administrativo, evaluar el marco legal relacionado con la empresa, interpretar información contable y financiera para la toma de decisiones gerenciales,)

ii) el proceso de monitoreo que los docentes deben desarrollar mediante las tareas duales, las cuales deben estar estrechamente relacionadas con los contenidos de cada módulo y las necesidades de las PYMES de la provincia. En este momento del proceso de formación, los docentes deben colaborar directamente con el monitor, discute los objetivos de la tarea que debe desarrollar el estudiante, los métodos que puede emplear y los resultados específicos de la tarea. El monitor verifica que el estudiante cumpla la secuencia establecida para el desarrollo de la tarea, define el tiempo para la ejecución y discute los errores cometidos en el desarrollo de la misma. Entre las tareas duales que pueden presentarse se encuentra el diagnóstico de las principales insuficiencias que se presentan en los sistemas de gestión de las empresas vinculadas a la formación dual, este tipo de tarea garantiza que el estudiante aplique las técnicas de gestión aprendidas en el proceso de formación en la institución educativa y que la empresa pueda perfeccionar su sistema de gestión en función de lograr un mejor desempeño.

Cuarta etapa. Seguimiento y asesoría: en esta etapa el objetivo es evaluar los resultados alcanzados en las organizaciones involucradas en la formación dual y acompañar al monitor de manera personalizada para que el formando cumpla con sus objetivos de aprendizaje. El docente reúne toda la información sobre la asistencia y calificación del estudiante, identifica y corrige irregularidades que se hayan presentado en el proceso de aprendizaje y, además, recopila la información necesaria para la actualización curricular de la carrera. Es importante que se evalúe no solo los conocimientos y habilidades que debe demostrar el estudiante, sino también los valores asociados a la actividad laboral que debe desarrollar en el proceso de formación en la empresa.

Uno de los elementos más importante en la formación dual es la conformación de la malla curricular para lograr formar las competencias que se requieren en función del formando y de las PYMES donde se desarrolla el proceso de enseñanza aprendizaje, a continuación, se expone en la tabla 1 el ejemplo para las carreras de ciencias empresariales. En este proceso de construcción de la malla curricular, deben incluirse los resultados del proceso de retroalimentación de la tercera etapa. 
Tabla 1: Ejemplo de conformación de la malla curricular para el proceso de formación dual en ciencias empresariales.

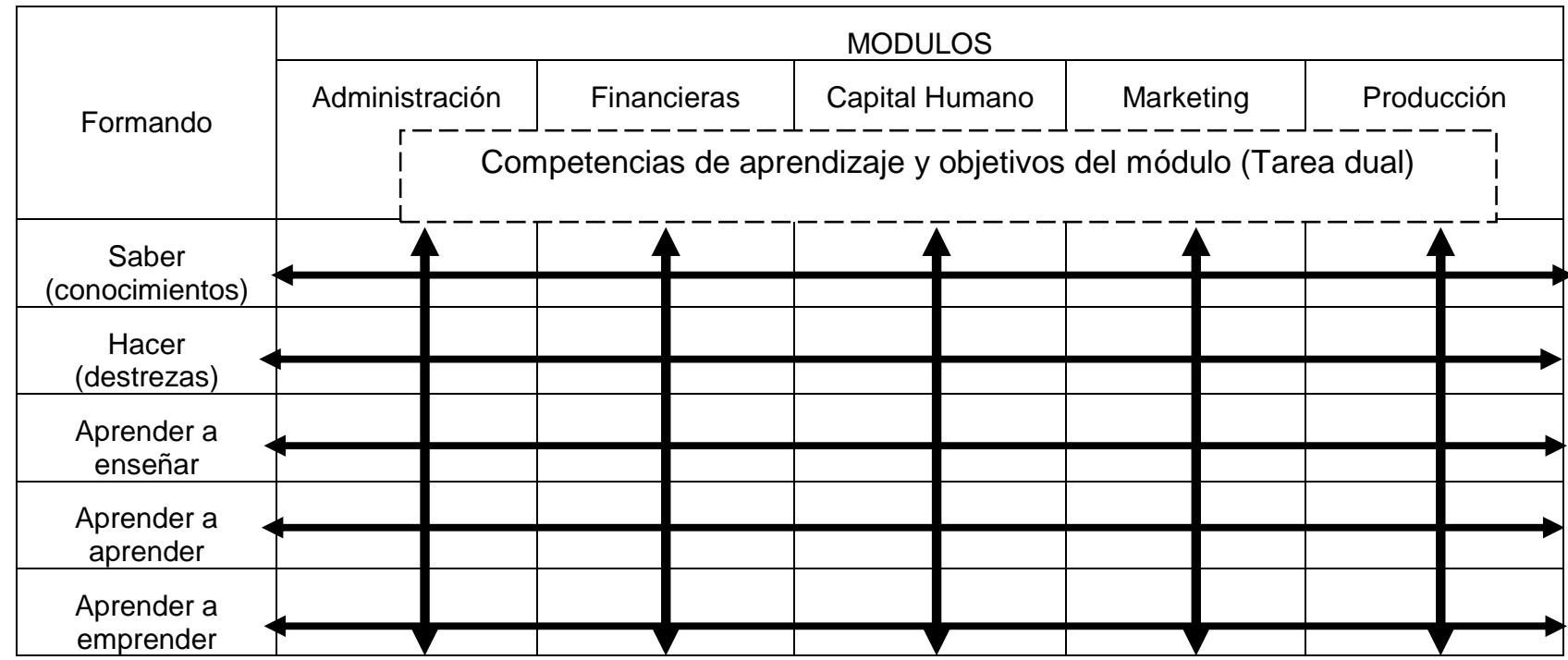

Beneficios de la formación dual para los estudiantes, las Pymes de Tungurahua y la FCADM.

Estudiantes: desarrollan competencias sociales al interactuar con los miembros de las empresas donde se vinculan; conocen de manera temprana su futuro profesional y por tanto pueden lograr mayor motivación por la carrera; se familiarizan con el mercado laboral y conocen las particularidades de las empresas de la provincia; vinculan la teoría con la práctica en las empresas donde en futuro pueden desempeñarse; adquieren valores como puntualidad, responsabilidad y motivación por el trabajo; y tienen una mayor preparación para insertarse en el mercado laboral, por lo que se puede incrementar el nivel de inserción laboral que en la actualidad es bien baja.

Pymes de Tungurahua: se aprovecha el aporte de los estudiantes a la empresa; las empresas pueden contar con empleados mucho mejor preparados, aspecto que es una insuficiencia en la actualidad en la provincia; se logra reducir los costos de formación de nuevos trabajadores; mayor vinculación con la universidad para transferir conocimientos en función de solucionar los problemas de las empresas; se mejora la imagen de la empresa al demostrar su compromiso con la educación de las nuevas generaciones

FCADM: la facultad logra mayor vinculación con las empresas y el mercado laboral; se desarrolla una oferta formativa ajustada a las necesidades del desarrollo del sector empresarial de la provincia; se vinculan a los profesionales del sector empresarial al proceso de formación de los estudiantes y por tanto existe una mayor vinculación teoría y práctica en el aprendizaje; se actualizan los contenidos de las asignaturas y malla curricular ajustada a la realidad del mercado laboral.

\section{CONCLUSIONES}

La formación dual no ha tenido aplicaciones importantes en las universidades ecuatorianas, sin embargo, es una alternativa viable para lograr elevar la calidad de la formación de los profesionales en el área de las ciencias empresariales que garantice una mejor preparación para su inserción al mercado laboral.

El estudio de inserción laboral realizado a los graduados de las carreras de Marketing y Gestión de Negocios y Organización de Empresas de la Facultad de Ciencias Empresariales de la Universidad Técnica de Ambato evidenció que la mayoría de los graduados se encuentran empleados pero no vinculados a la profesión estudiada, sólo una pequeña minoría (alrededor del 20\%) han creado emprendimientos, igualmente la mayoría consideran que no se aprovecha el proceso de formación de la carrera para integrarse al sector empresarial y que la formación dual puede ayudar a prepararlos mejor para la inserción laboral.

Los empresarios de las PYMES de la provincia Tungurahua manifestaron conocer las carreras que se estudian en la Facultad de Ciencias Administrativas, pero menos del $60 \%$ de estos empresarios han realizado actividades vinculadas a estas carreras, además, consideran que la calidad de los graduados no es buena y que sería muy provechoso desarrollar la formación dual entre la universidad y sus empresas.

La estrategia propuesta para la implementación de la formación dual en las PYMES de la provincia Tungurahua contribuirá a mejorar la preparación de los graduados de las carreras de Marketing y Gestión de Negocios y Organización de Empresas de la Facultad de Ciencias Empresariales de la Universidad 
Técnica de Ambato para su inserción laboral y además a perfeccionar la gestión de los procesos de estas empresas.

\section{REFERENCIAS}

Adam, S. Mohamad, R. y M. Ruhizan, Industry Involvement Conceptual Framework for Collaboration of National Dual Training Systems (NDTS) in Malaysia. Sains Humanika, 9(2), 33-41 (2017)

Aguilar, J. y C. Bermúdez, Business-University Links. Innovation for the diversification of cocoa markets. RICEA Revista Iberoamericana de Contaduría, Economía y Administración, 4(7), 103-121 (2016)

Álvarez, J. y A. Romero, La empleabilidad de los graduados universitarios en el contexto latinoamericano. Realidades de UNIANDES. Ecuador. Revista Atenas, 4 (32), 1-15 (2015)

Arias, M.F., La vinculación Universidad-Empresa-Estado, ¿es necesaria? La percepción de académicos argentinos. Revista Iberoamericana de Ciencias Empresariales y Economía, 4(4), 11-30 (2014)

Carranza, A.G., Acosta, M.G., y R. E. Pulido, Dual training model of the National Technological Mexico. Revista de Investigación en Educación, 14(2), 170-183 (2016)

Casalet, M., Las relaciones de colaboración entre la universidad y los sectores productivos: una oportunidad a construir en la política de innovación. Dilemas de la Innovación en México: Dinámicas sectoriales, territoriales e institucionales. Guadalajara, Jal.: COLEF-CONACYT, 109-142 (2012)

Castillo, J. A., Guerra, L. A., y P. V., Zárate, La educación dual y el programa académico de ingeniería industrial en los institutos tecnológicos. ANFEl Digital, 2(3), 1-9 (2016)

Coiduras, J., París, G., Torrelles, C., y X., Carrera, La evaluación de competencias en una experiencia de formación dual de maestros: diferencias y semejanzas entre tutores de escuela y de universidad. Estudios pedagógicos (Valdivia), 40 (ESPECIAL), 29-48 (2014)

Hernández, R., y L. Y. Goe, La formación dual en Colombia. El caso de la Fundación Universitaria de la Cámara de Comercio de Bogotá Uniempresarial: los desafíos actuales en la percepción de egresados y empresarios. Revista Científica General José María Córdova, 13(15), 145-181 (2015)

Herrera, F., Guzmán, J., y G. Tinoco, Modalidad de estudio dual, un nuevo modelo educativo en la formación de ingenieros. ANFEI Digital, (2), 21-30 (2015)

Kupffer, J., Formación Profesional Dual en Latinoamérica. El sistema Dual y su transferencia. Cámara de Comercio e Industria Boliviano Alemana, 14-19, La Paz, Bolivia (2015)

León, C., Análisis de la integración de teoría y la práctica de la disciplina de administración de operaciones en la formación de administradores de empresas, reflexión desde lo académico y laboral. Universidad y Sociedad, 8(1), 50-58 (2016)

Llisterri, J., Gligo, N., Homs, O., y D. Ruiz, Educación técnica y formación profesional en América Latina, El reto de la productividad. Serie Políticas Públicas y Transformación Productiva, (13), 1-87 (2014)

Marsollier, R., y M., Aparicio, Universidad, empleo y expectativas de logro en el mundo del trabajo. Revista Enfoques, 26(2), 87-98 (2016)

Mato, D., Contribución de experiencias de vinculación social de las universidades al mejoramiento de la calidad académica y factores que limitan su desarrollo y valoración institucional. Revista da Avaliação da Educação Superior, 18(1), 151-180 (2013)

Montoya, J. H., Formación por competencias: racionalidades e implicaciones en el mundo del trabajo. Revista Universitas Odontológica, 31(66), 75-82 (2012)

Montoya, R., Francisco, J., y J. Aguilar, La Relación Universidad-Empresa en las Prácticas Empresariales: un Modelo Conceptual desde las Técnicas de Generación de Ideas. Journal of technology management \& innovation, 8, 196-208 (2013)

Muñoz, I.A., La Formación Dual y su fundamentación curricular. Revista Educación, 32(1), 15-24 (2008) 
Novelo, J. N., Solís, G. R., y E. C. Coenhour, Vinculación universidad-empresa por un mundo más sustentable. Revista Electrónica sobre Tecnología, Educación y Sociedad, 1(6), 1-11 (2016)

Organización Internacional del Trabajo (OIT). Panorama Laboral de América Latina y El Caribe 2015, $1^{\circ}$ Ed., pp. 28-47, Oficina Regional para América Latina y el Caribe, Lima, Perú (2015)

Quispe, A., Victorino, L., y R. Á. Atriano, Vinculación de Instituciones de Educación Agrícola Superior (IEAS) con sectores productivos: El caso de la residencia profesional en el Instituto Tecnológico del Altiplano de Tlaxcala (ITAT). Revista de la educación superior, 43(170), 135-152 (2014)

Rodríguez, M. J., Vicente, J. L., y G. G. Siles, An experience in university-enterprise relations: the cogempleo project in the technological foundation campus of Algeciras. Educación XX1, 19(1), 201-220 (2016)

Ruiz, S.L.R., y C. Acevedo, Vinculación de la academia con el sector productivo. Revista Matices Tecnológicos, 5 (2), 37-42 (2015)

Tokeran, M. F. y R. Wahid, the Development of Work-Process-Orientation Training Material for National Dual Training System (NDTS) Implementation. Advanced Science Letters, 23(2), 953-957 (2017)

UNESCO. Conferencia Mundial sobre la Educación Superior - 2009: La nueva dinámica de la educación superior y la investigación para el cambio social y el desarrollo, 1-9, París, Francia 5 al 8 de julio (2009)

Vega, J., Manjarrez, H., Castro, F. y L., Fernández, Las relaciones universidad-empresa: tendencias y desafíos en el marco del Espacio Iberoamericano del conocimiento. Revista Iberoamericana de Educación, 57, 109-124 (2011)

Velho, L., Velho, P. V., y A. Davyt, Las políticas e instrumentos de vinculación Universidad-Empresa en los países del MERCOSUR. Educación superior y sociedad, 9(1), 51-76 (2010)

Vicente, M.E., Educación y trabajo en ciencias de la educación: Aportes desde el estudio de las trayectorias profesionales de sus graduados. DOI: 10.4067/S0718-50062012000600006. Revista Formación Universitaria, 5(6), 51-62 (2012)

Yahaya, N., Sattar, R., y Mohamad, R., Exploring the Authentic Teaching and Learning Experience of the Apprentices of the National Dual Training System. International Journal of Academic Research in Business and Social Sciences, 7(3), 690-703 (2017)

Zholdasbekova, S., Nurzhanbayeva, Z., Mavedov, R., Saipov, A., Zhiyentayeva, B., y A. Tlemissova, Didactic Conditions of Improvement of Pedagogical Personnel Training at Higher Education Institutions to Dual Education in the System of VET. International Journal of Environmental \& Science Education, 11(18), 12345-12349 (2016) 